\title{
Método para la formulación de hormigones de fibras metálicas
}

\author{
PEDRO SERNA ROS \\ Departamento de Materiales de Construcción E.T.S.I.C.C.P. \\ Universidad Politécnica. Valencia
}

\section{$R E S U M E N$}

En los trabajos que presentamos nos hemos planteado el objetivo de determinar un método práctico de dosificación de hormigones de fibras. Para ello partiremos, como en cualquier problema de dosificación, de unas posibilidades de suministro que nos condicionarán la naturaleza de los áridos a utilizar, de unas limitaciones técnicas impuestas por los aditivos, y de unas necesidades de puesta en obra que nos fijarán la trabajabilidad del hormigón.

La primera parte de nuestro trabajo se reduce a determinar, a partir de la dosificación en cemento fijada inicialmente, y para cada contenido en fibras propuesto, los porcentajes óptimos de cada uno de los áridos y la relación agua/cemento.

En la segunda parte desarrollaremos el estudio de los limites admisibles del contenido en fibras, para evitar la formación de "pelotas de fibras".

\section{$S U M M A R Y$}

In the work we are presenting, we have set ourselves the target of determining a practical method for ascertaining the dosage of fibrous concrete. To do this, as in any problem regarding dosage, we shall start from the possibilities of supply, which will be conditioned by the nature of the aggregates to be used, of certain technical restrictions imposed by the admixtures and of the carrying out requirements which will set the concrete workability.

The first part of our study amounts to determining the optimal percentages for each aggregate and the water-cement ratio, starting with the dosage of cement established initially and for each fibre content proposed.

In the second part, we shall develop the study of acceptable limits of fibre content, in order to prevent 'balls of fibre' from forming.

\section{INTRODUCCION}

La adición de una cierta cantidad de fibras a la matriz de un hormigón no exige la utilización de materiales especiales. Se podrá utilizar el mismo cemento, los mismos áridos, así como el agua y los aditivos. Sin embargo, para el mejor aprovechamiento de las fibras, se exigirá un estudio delicado de la dosificación para afrontar los dos principales problemas que se acusan en el hormigón de fibras:

- la disminución de la trabajabilidad (1), (2),

- el riesgo de formación de "pelotas de fibras" debido a una mala distribución de éstas (segregación especifica de las fibras).

Para evitar el riesgo de segregación DEHOUSSE (3) aconseja limitar a 0,5 el cociente entre el peso del árido mayor de $5 \mathrm{~mm}$ y el peso del árido total, mientras que la USCOLD Concrete Committee (4) propone granulometrias límite en función del tamaño máximo del árido. Asi mismo se suelen utilizar dosificaciones en cemento elevadas (de 400 a $550 \mathrm{~kg} / \mathrm{m}^{3}$ ). 
Con esta misma orientación MOENS (5) propone limitar el contenido en fibras por medio del parámetro $V_{\mathrm{f}} l / d<C_{\mathrm{m}}$, siendo:

$V_{\mathrm{f}}$ el porcentaje, en volumen, de fibras,

$l / d$ su longitud relativa,

$C_{\mathrm{m}}$ un coeficiente que depende de la granulometria utilizada.

Si bien estas limitaciones pueden servir de base para la formulación de un hormigón "tipo fibras", no son suficientes para optimizar su composición ni para valorar el riesgo de segregación.

Por otra parte, la reducción de la trabajabilidad se ha solucionado generalmente por medio de la adición de superplastificantes. Este método de actuar puede utilizarse en casos concretos, pero no siempre es una solución aceptable ya que cualquier aditivo tiene limitada su dosificación.

En los trabajos que presentamos nos hemos planteado el objetivo de determinar un método práctico de dosificación de hormigones de fibras. Para ello partiremos, como en cualquier problema de dosificación, de unas posibilidades de suministro que nos condicionarán la naturaleza de los áridos a utilizar, de unas limitaciones técnicas impuestas por los aditivos, y de unas necesidades de puesta en obra que nos fijarán la trabajabilidad del hormigón.

La primera parte de nuestro trabajo se reduce a determinar, a partir de la dosificación en cemento fijada inicialmente, y para cada contenido en fibras propuesto, los porcentajes óptimos de cada uno de los áridos y la relación agua/cemento.

En la segunda parte desarrollaremos el estudio de los límites admisibles del contenido en fibras, para evitar la formación de "pelotas de fibras".

La parte experimental de las investigaciones que presentamos ha sido realizada en el "Laboratoire Central des Ponts et Chaussées" de Paris (6). Se han estudiado únicamente fibras metálicas Dramix $50 / 50$ (50 $\mathrm{mm}$ de longitud y $0,5 \mathrm{~mm}$ de diámetro) fabricadas por la sociedad BEKAERT.

\section{COMPOSICION OPTIMA DE UN HORMIGON DE FIBRAS}

\subsection{Bases de dosificación}

Para determinar la composición de un hormigón de fibras hemos aceptado los principios del método de dosificación de BARONLESAGE (7) que propone que, dentro de los límites de las composiciones normales de hormigón:

- Fijadas las dosificaciones de agua y de cemento, el hormigón más fácil de poner en obra es el más compacto, y su esqueleto mineral es el óptimo.

- La composición óptima de los áridos es independiente de las dosificaciones de agua y de cemento.

Por nuestra parte hemos supuesto que la introducción de fibras no modifica la validez de estos principios.

Con estas hipótesis el estudio de las dos variables (composición granulométrica y relación agua/cemento) puede realizarse separadamente en dos fases: 


\section{1. ${ }^{\mathrm{a}}$ FASE. Dosificación de los áridos}

Se fijará una relación agua/cemento y se estudiará la trabajabilidad del hormigón, variando únicamente los porcentajes $\left(p_{1}, p_{2}\right)$ de los áridos caracterizados por sus módulos granulométricos $\left[m_{1}, m_{2}\right.$; el módulo granulométrico de la mezcla puede calcularse por $\left.m=\left(m_{1} p_{1}+m_{2} p_{2}\right) / 100\right]$. La composición granulométrica que obtenga la máxima trabajabilidad será la composición óptima. Según el segundo de los principios enunciados esta granulometria será la óptima cualquiera que sea la dosificación definitiva de agua.

Con estas bases la obtención de la composición granulométrica óptima puede conseguirse por medio de curvas análogas a la representada en la figura 1.

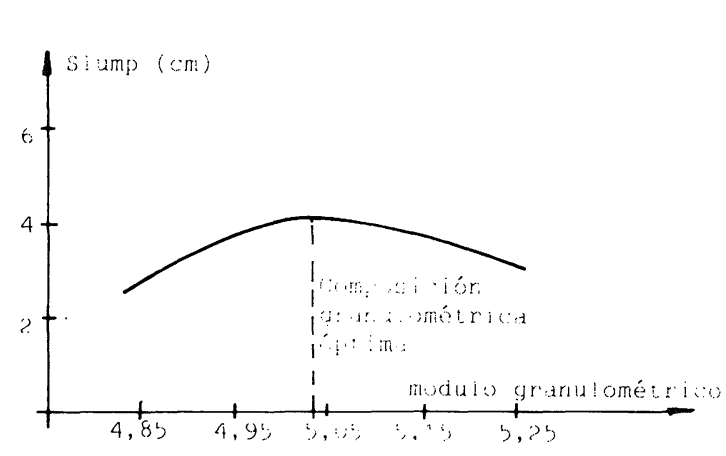

Fig. 1.-Determinación de la composicion granulométrica óptima.

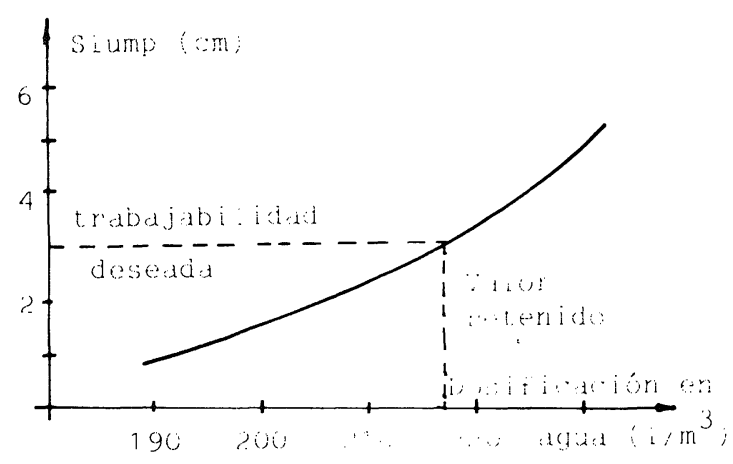

Fig. 2.--Determinación de la dosificación del agua.

\section{2. ${ }^{a}$ FASE. Relación agua/cemento}

Variando la dosificación en agua del hormigón, cuyo esqueleto mineral ha sido optimizado, elegiremos aquella que presente la trabajabilidad deseada (figura 2).

El método de dosificación se reduce, por consiguiente, a la realización de estudios de trabajabilidad. El ensayo que hemos utilizado en nuestras investigaciones ha sido el del asiento en el cono de Abrams (Slump) por ser el de mayor difusión.

\subsection{Determinación de la composición granulométrica óptima en función de la dosificación en fibras}

Siguiendo el método anteriormente descrito hemos estudiado las modificaciones que la presencia de fibras induce en la composición óptima de las 5 series de hormigón cuyos constituyentes se indican en el cuadro 1 .

Las curvas granulométricas de los áridos empleados se indican en la figura 3.

Para cada serie de hormigón hemos estudiado 5 dosificaciones de fibras (0-0,5-1-1,5 y $2 \%$ en volumen) y ajustado la relación arena/árido total que optimiza la composición. Se han realizado en total 150 hormigonadas y 300 ensayos de asiento en el cono de Abrams.

El conjunto de los resultados se indican en el cuadro 2, y los correspondientes a las series 1 a 4 se representan en la figura 4. 


\section{CUADRO 1}

Constituyentes de los hormigones utilizados en el estudio de las composiciones granulares óptimas

\begin{tabular}{|c|c|c|c|c|}
\hline Serie & $\begin{array}{l}\text { Cemento } \\
\text { CPA 55 } \\
\left(\mathrm{kg} / \mathrm{m}^{3}\right)\end{array}$ & $\begin{array}{c}\text { Plastif. } \\
\text { Melment } \\
\left(\mathrm{kg} / \mathrm{m}^{3}\right)\end{array}$ & Arena (tipo) & Arido grueso (tipo) \\
\hline 1 & 400 & 6 & $\begin{array}{l}\text { Composición de: } \\
-13 \% \text { arena de Fontainebleau } \\
-32 \% 0 / 1 \text { silico-calc. rodado } \\
-55 \% 1 / 4 \text { silico-calc. rodado }\end{array}$ & $4 / 10$ silico-calc. rodado \\
\hline 2 & 400 & 6 & $0 / 5$ silico-calc. machacado & 4/10 silico-calc. rodado \\
\hline 3 & 400 & 6 & & $5 / 20$ silico-calc. machacado \\
\hline 4 & 400 & 6 & \multirow{3}{*}{$0 / 5$ silico-calc. machacado } & \multirow{3}{*}{$8 / 14$ pórfido } \\
\hline $5 \mathrm{~A}$ & 325 & 4.9 & & \\
\hline $5 \mathrm{~B}$ & 475 & 7.1 & & \\
\hline
\end{tabular}

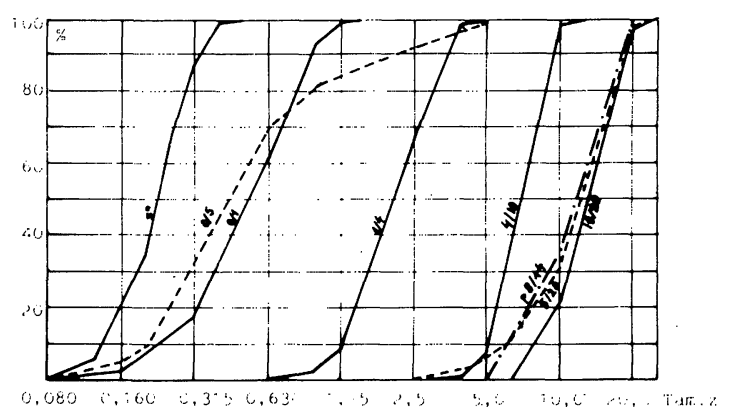

Fig. 3.-Aridos de base utilizados.

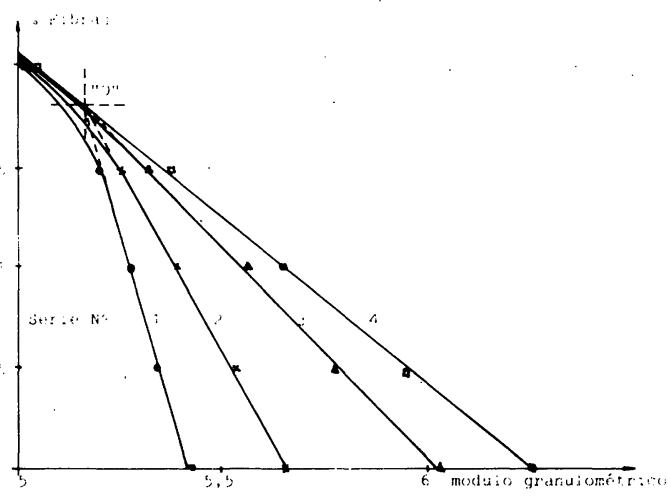

Fig. 4.-Composición del hormigón óptimo, en función del contenido en fibras.

C UADRO 2

Resultados de los ensayos para la determinación de la composición granulométrica óptima

\begin{tabular}{|c|c|c|c|c|}
\hline \multirow{2}{*}{$\begin{array}{c}\text { \% de fibras } \\
\text { (en volumen) }\end{array}$} & \multicolumn{4}{|c|}{ Módulo granulométrico óptimo } \\
Serie N.
\end{tabular}


Se comprueba que, para cada serie de hormigón y para valores de dosificación en fibras inferiores a $1,5 \%$, los valores del módulo granulométrico obtenidos están alineados y que todas las rectas concurren en el punto " 0 " correspondiente a un módulo granulométrico de 5,16 y a una dosificación en fibras de $1,8 \%$.

Estos resultados nos permiten deducir que, conociendo la granulometria de dos áridos, podemos calcular, para cualquier dosificación en fibras prefijada, las proporciones de cada uno de ellos necesarias para obtener el hormigón óptimo. Para ello es suficiente determinar la dosificación óptima del hormigón sin fibras y calcular su módulo granulométrico $m_{\mathrm{io}}$; con este valor determinamos en el eje de abscisas de la figura 4 el punto B. Uniendo este punto con el punto " 0 " obtendremos la recta que nos permite calcular el módulo granulométrico óptimo $m_{\mathrm{ip}}$ correspondiente a cualquier porcentaje en fibras $p_{\mathrm{i}}$. Analíticamente este valor puede obtenerse por la fórmula:

$$
m_{\mathrm{ip}}=m_{\mathrm{io}}-\frac{m_{\mathrm{io}}-5,16}{1,8} p_{\mathrm{i}}
$$

El análisis de la figura 4 muestra que, cuanto mayor es el módulo granulométrico del hormigón sin fibras, la introducción de éstas exige una mayor disminución de dicho módulo, es decir una mayor reducción del contenido en árido grueso. En cambio, en el caso de hormigones finos, la introducción de fibras hasta porcentajes de $1,5 \%$ en volumen, apenas modifica el módulo granulométrico óptimo del hormigón.

Los resultados de los ensayos correspondientes a las series 4 y 5 , en las que la única variable era la dosificación en cemento, nos han servido para verificar que si el contenido en cemento se mantiene dentro de los limites corrientes (de 325 a $475 \mathrm{~kg} / \mathrm{m}^{3}$ ), éste no influye en la dosificación óptima de los áridos.

\section{DETERMINACION DEL CONTENIDO MAXIMO EN FIBRAS. LIMITES DE TRABAJABILIDAD}

La segunda. fase del estudio de la composición de un hormigón de fibras consiste en la determinacion del contenido en agua que conduce a la obtención de la trabajabilidad exigida por las condiciones de puesta en obra.

La experiencia. nos ha demostrado que este proceso, sencillo en su planteamiento, puede presentar complicaciones adicionales. Hemos podido comprobar que, en algunos casos en los que la composición granulométrica fue optimizada, con una dosificación en agua determinada y presentando el hormigón un aspecto homogéneo, al aumentar la relación agua/cemento se observó la aparición de "pelotas de fibras", es decir una segregación específica de éstas.

Este hecho indica que el límite de dosificación en fibras no depende únicamente de la composición granulométrica del hormigón, sino también de la relación agua/cemento, o más bien de la trabajabilidad del hormigón. Podemos, por consiguiente plantear el problema del contenido máximo de fibras de la siguiente manera: para una determinada composición granulomérica y para una dosificación en fibras prefijada, determinar la trabajabilidad máxima del hormigón que no provoca la segregación específica de las fibras (trabajabilidad límite).

El procedimiento para resolver este problema será el de fijar la dosificación del hormigón, a excepción del contenido en agua, y variar la relación agua/cemento. Para cada valor de esta variable estudiaremos la trabajabilidad del hormigón, obteniendo curvas similares a la representada en la figura 5. Supondremos que se ha sobrepasado el limite superior admisible de 
trabajabilidad cuando se observe la aparición de "pelotas de fibras" y retendremos como valor limite la trabajabilidad del hormigón inmediatamente anterior del que provoca la segregación especifica de las fibras.

Hemos estudiado, de este modo, la influencia conjunta de la composición granulométrica y del contenido en fibras sobre la trabajabilidad limite en los hormigones indicados en el cuadro 3 , en el que se precisan los resultados de los ensayos, que quedan representados en la figura 6. Por interpolación hemos representado las curvas de isotrabajabilidad que nos permitirán conocer, para una composición determinada (de áridos y fibras) el riesgo de segregación en función de la trabajabilidad deseada.

Observando estas curvas podemos concluir:

- En los hormigones con módulo granulométrico bajo se pueden utilizar dosificaciones altas en fibras (hasta $1 \%$ en volumen) sin precauciones particulares. Se puede, incluso, llegar a $2 \%$ o más siempre que se controle con precisión el contenido en agua, ya que una pequeña variación de la trabajabilidad puede provocar la segregación de las fibras. Este hecho justifica el porque las formulaciones de "hormigones tipo fibras" utilizadas normalmente limitan el tamaño máximo de árido a $10 \mathrm{~mm}$.

- En hormigones con módulo granulométrico elevado se debe limitar el contenido en fibras a 0,5 ó $0,6 \%$ en volumen. En estos casos es indispensable un control minucioso de esta dosificación ya que un exceso de fibras provocaría el paso de la zona de hormigones homogéneos, para cualquier trabajabilidad, a la zona de segregación de las fibras.

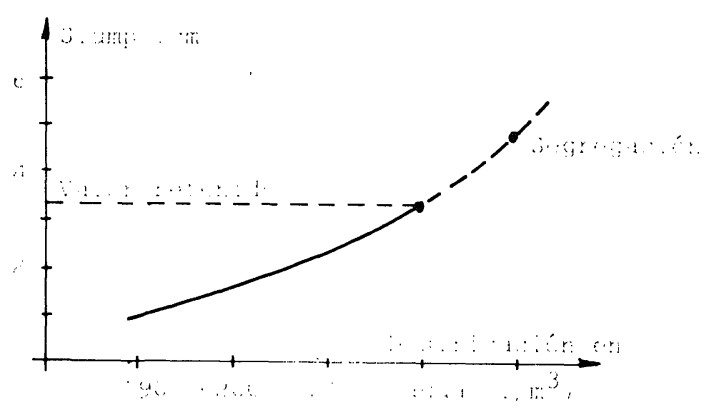

Fig. 5.-Determinación de la trabajabilidad limite.

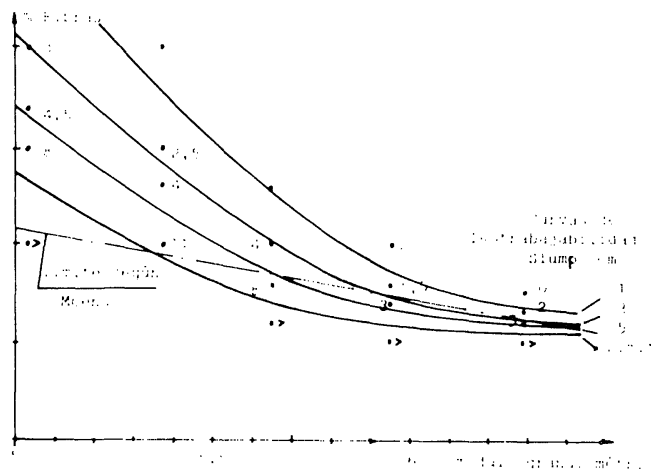

Fig. 6. - Limites de contenido en fibras segun la trabajabilidad.

\section{UADRO 3}

Resultados de los ensayos de trabajabilidad limite sin segregación de fibras Slump (cm)

\begin{tabular}{|c|c|c|}
\hline $\begin{array}{c}\text { Módulo } \\
\text { granulométrico }\end{array}$ & $\begin{array}{l}\% \text { de fibras } \\
\text { (en volumen) }\end{array}$ & $\begin{array}{c}\text { Trabajabilidad } \\
\text { limite }\end{array}$ \\
\hline 5,02 & $\begin{array}{l}1 \\
1,5 \\
1,7 \\
2\end{array}$ & $\begin{array}{l}> \\
8 \\
4,5 \\
3\end{array}$ \\
\hline 5.40 & $\begin{array}{l}1 \\
1,3 \\
1,5 \\
2\end{array}$ & $\begin{array}{l}11 \\
4 \\
2,5 \\
0\end{array}$ \\
\hline 5,65 & $\begin{array}{l}0,6 \\
0.8 \\
1 \\
1,3\end{array}$ & $\begin{array}{l}> \\
8 \\
4 \\
1\end{array}$ \\
\hline 5,95 & $\begin{array}{l}0,5 \\
0,7 \\
0,8 \\
1\end{array}$ & $\begin{array}{l}> \\
3 \\
1.5 \\
0\end{array}$ \\
\hline 6,30 & $\begin{array}{l}0.5 \\
0.6 \\
0,7 \\
0.8\end{array}$ & $\begin{array}{l}> \\
5 \\
2 \\
0\end{array}$ \\
\hline
\end{tabular}




\section{ABACO PARA LA FORMULACION DE HORMIGONES DE FIBRAS}

Todo lo expuesto anteriormente puede recopilarse en un ábaco que sería la yuxtaposición de las figuras 4 y 6 y que queda representado en la figura 7 .

La utilización de este ábaco nos permitirá, para dosificaciones normales en cemento, determinar las composiciones granulométricas óptimas del hormigón en función del contenido en fibras; trazaremos para ello la recta OB correspondiente. La curva de isotrabajabilidad que pase por el punto característico $\mathrm{C}$ indicará la trabajabilidad limite sin segregación específica de las fibras.

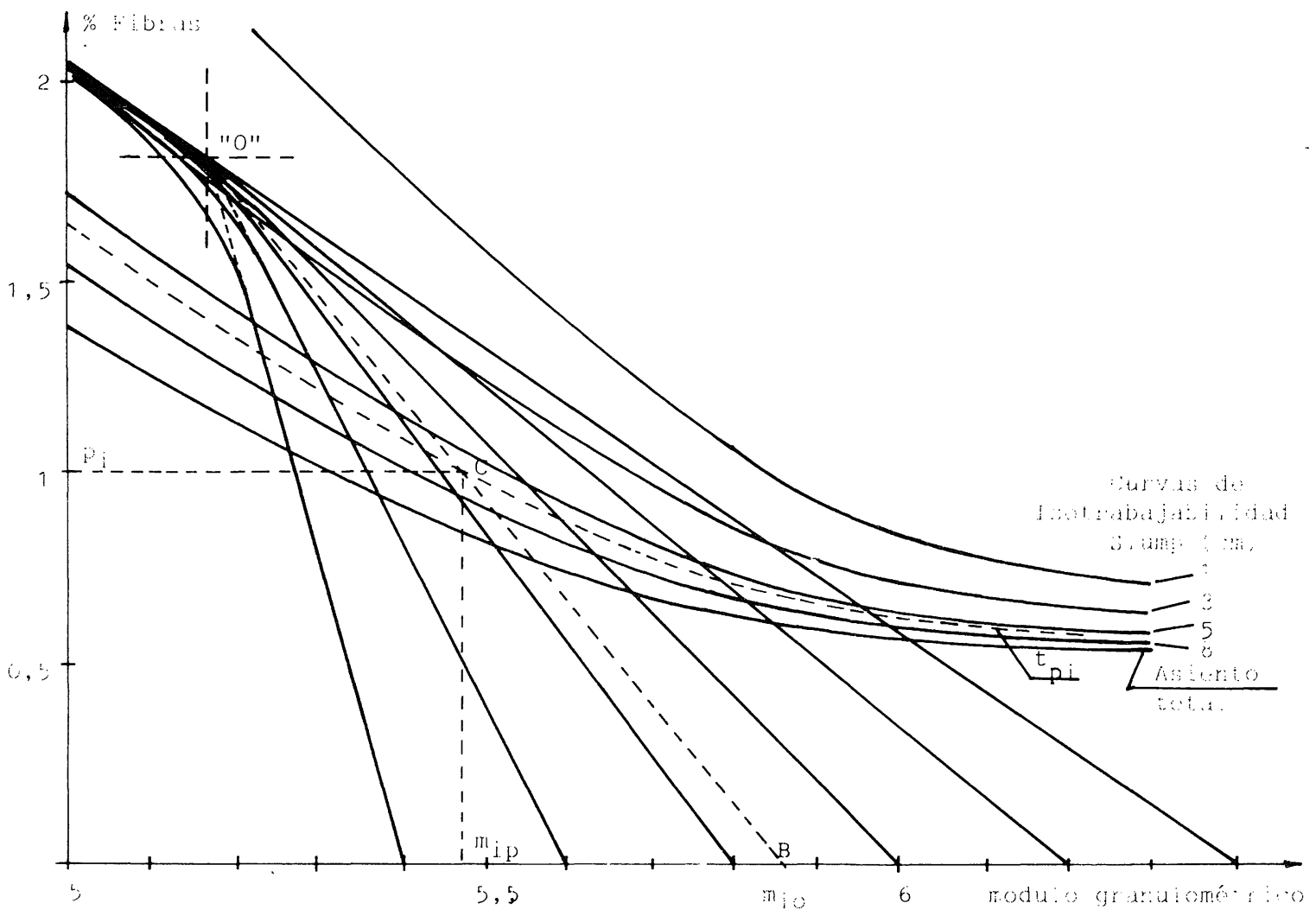

Fig. 7.-Abaco para la formulacion de hormigones de fibras

\section{B I B L I O G R A F I A}

(1) SWAMY, R. N.; MANGAT, P. S.: Influence of fiber geometrie on the properties of steel fiber reinforced concrete. USA-Cement Concr. Res. Vol. 4, n.० 3, pp. 451-465. Mai 1974.

(2) EDGINGTON, J.; HANNANT, D. J.; WILliAM, R. I. T.: Steel fibre reinforced concrete. G. B. Building Res. Est. Current papet C. P. 69/74. 19 p. July 1974.

(3) DEhOUSSE, N.; LEJEUNE, A.; CUYKENS, T.: Les mortiers et bétons reforcés de fibres d'acier. Bruxelles. C.S.T.C. revue 2. pp. 2-4. èté 1974 .

(4) USCOLD Concrete Committee: State of the art report on fiber reinforced concrete. Junc 1981.

(5) MOENS, J. E. C.: Steel fiber concrete mix proportioning. Philadelphie. Paper presented at the ACI convention. Mars 1976.

(6) SERNA, P.: Una aportación al conocimiento del hormigón de fibras metálicas en su conocimiento, propiedades y comportamiento mecánico. Tésis doctoral presentada en la E.T.S.I.C.C. y P. de Valencia. Mayo 1984.

(7) BARON, J.; LESAGE, R.: La composition du betón hydraulique du laboratoire au chantier. F. Rapport de Recherche L.P.C. n." 64. Decembre 1976. 